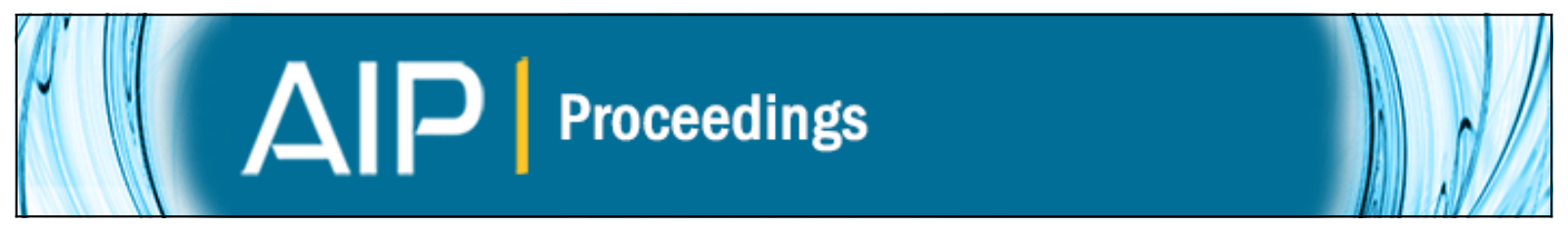

\title{
Study of EEG Brain Maturation Signals with Multifractal Detrended Fluctuation Analysis
}

A. Figliola, E. Serrano, J. A. P. Rostas, M. Hunter, and O. A. Rosso

Citation: AIP Conference Proceedings 913, 190 (2007); doi: 10.1063/1.2746746

View online: http://dx.doi.org/10.1063/1.2746746

View Table of Contents: http://scitation.aip.org/content/aip/proceeding/aipcp/913?ver=pdfcov

Published by the AIP Publishing

Articles you may be interested in

Multifractal detrended moving average analysis for texture representation

Chaos 24, 033127 (2014); 10.1063/1.4894763

Dynamics of cellular response to hypotonic stimulation revealed by quantitative phase microscopy and multifractal detrended fluctuation analysis

Appl. Phys. Lett. 101, 203702 (2012); 10.1063/1.4766922

Common multifractality in the heart rate variability and brain activity of healthy humans

Chaos 20, 023121 (2010); 10.1063/1.3427639

Fluctuations in Neuronal Activity: Clues to Brain Function

AIP Conf. Proc. 780, 595 (2005); 10.1063/1.2036823

Mapping of Synaptic-Neuronal Impairment on the Brain Surface through Fluctuation Analysis

AIP Conf. Proc. 780, 541 (2005); 10.1063/1.2036810 


\title{
Study of EEG Brain Maturation Signals with Multifractal Detrended Fluctuation Analysis
}

\author{
A. Figliola*, E. Serrano ${ }^{\dagger}$, J. A. P. Rostas ${ }^{* *}$, M. Hunter ${ }^{\ddagger}$ and O. A. Rosso ${ }^{\S}$ \\ *Instituto del Desarrollo Humano, Universidad Nacional de General Sarmiento. \\ Juan Maria Gutierrez 1150, Los Polvorines, Pcia. de Buenos Aires, Argentina. \\ ${ }^{\dagger}$ Escuela de Ciencia y Tecnologia, Universidad Nacional de General San Martín. \\ M. Irigoyen 3100, San Martín, Pcia. de Buenos Aires, Argentina. \\ ${ }^{* *}$ Biomedical Sciences and Hunter Medical Research Institute, University of Newcastle. \\ University Drive, Callaghan, NSW 2308, Australia. \\ School of Behavioural Sciences and Hunter Medical Research Institute. University of Newcastle. \\ University Drive, Callaghan, NSW 2308, Australia. \\ $\S$ Instituto de Cálculo, Facultad de Ciencias Exactas y Naturales, Universidad de Buenos Aires. \\ Pabellon II, 1428 Ciudad Universitaria, Buenos Aires, Argentina.
}

\begin{abstract}
In this work, we have study the EEG signals of birds during the first 6 weeks of life. The aim of the article is to perform a quantitative analysis of the dynamical changes observed in these signals due to the brain maturation effects. The signals' long scaling behaviour is study by Multifractal Detrended Fluctuation Analysis (MFDFA). This method allows the multifractal characterization of these EEG nonstationary time series and characterize the different stage of bird brain maturation.
\end{abstract}

Keywords: EEG, brain maturation, multifractal analysis.

PACS: 05.45.Df, 87.19.La, 89.75.Fb

\section{INTRODUCTION}

Animal models provide an invaluable source of information in the testing of hypotheses that are difficult to corroborate by other experimental means. In present work, we revisit data analysis of scalp-applied recording electrodes which have been used to monitor changes in basal EEG patterns in chickens during posthatch development [1]. The motivation here is to study the development and maturation of brain function using the Multifractal Detrended Fluctuation Analysis (MFDFA) [2].

In recent years, the Detrended Fluctuation Analysis (DFA) [3] has been established as an important tool for the detection of long-range (auto-)correlations in time series and has become a widely used technique for the determination of (mono-)fractal scaling properties and the detection of long-range correlations in noisy, nonstationary time series. The DFA has successfully been applied to diverse fields. An alternative approach for the study of the multifractal behavior has been proposed by Stanley and co-workers [2]. It is based on a generalization of the DFA method and is know as Multifractal Detrended Fluctuation Analysis (MFDFA).

In this work, we characterized EEG signals of chicken, since the birds have weekly measues until six weeks posthatch. It is considered that in that period the chicken reach their neurological maturity. Using the MFDFA method we have been able to build the multifractal spectrum of each EEG time series corresponding to the analyzed weeks and perform a quantitative analysis of the brain maturation changes.

\section{EXPERIMENTAL DATA AND ARTIFACT REDUCTION}

Twenty four chickens (Gallus domesticus) reared from hatching were the subjects in this experiment. All birds had free access to food and water throughout the experiment. The birds were reared initially in incubation boxes and then transferred to holding cages maintained at a constant temperature $\left(21^{\circ} \mathrm{C}\right)$ with a $12: 12 \mathrm{hr}$ light:dark cycle.

Continuous EEG recordings $(0.1-100 \mathrm{~Hz}$ with a $50 \mathrm{~Hz}$ notch filter) were made using small $(6 \mathrm{~mm})$ gold cup electrodes attached to the scalp with collodion glue and filled with electrode gel. The signal was sampled at a rate of 


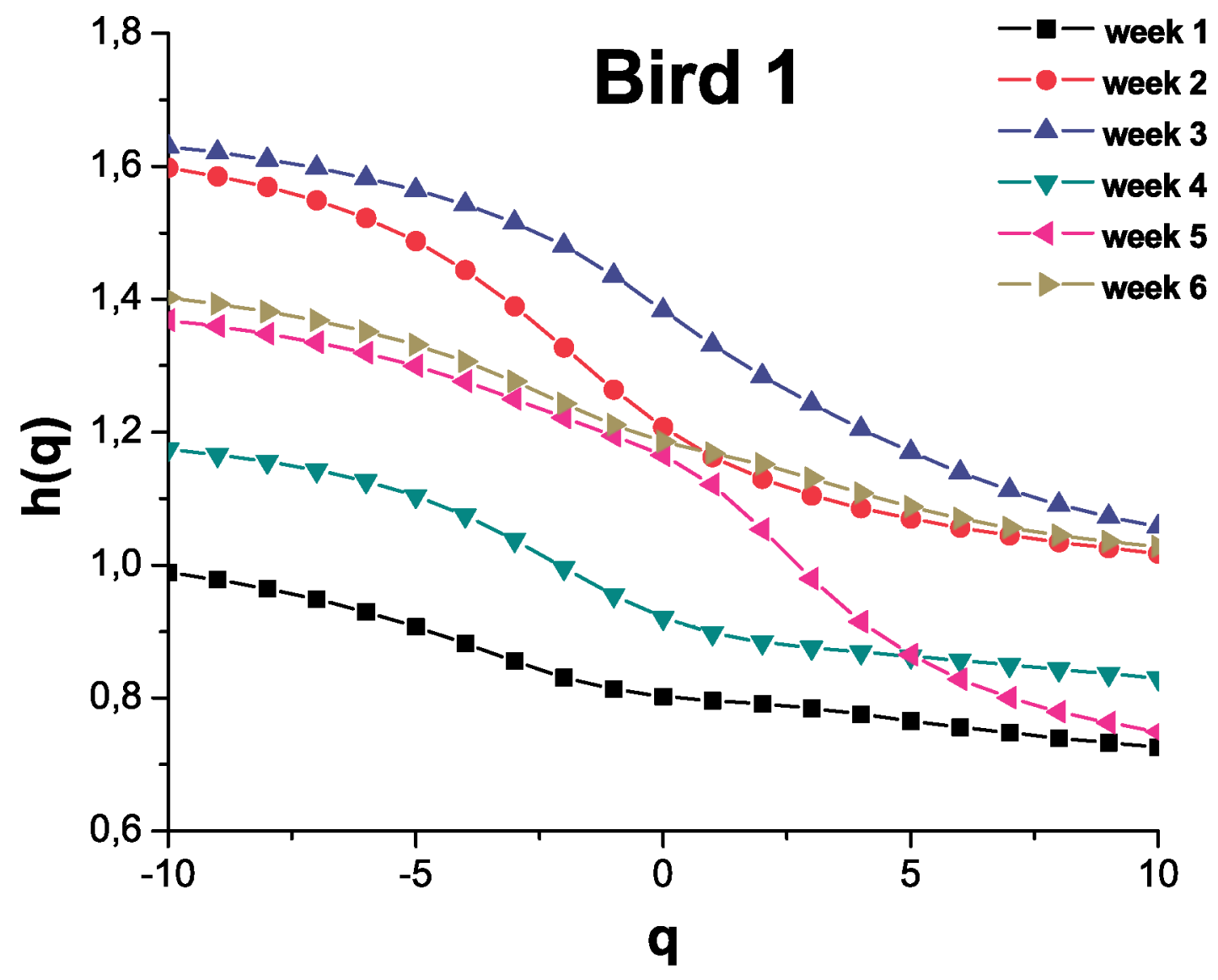

FIGURE 1. $H(q)$ corresponding to Bird $\# 1$ at frontal left electrode for the 6 weeks' posthatch.

$128 \mathrm{~Hz}$, passed through amplifiers, and stored directly on computer. The total length of each record is $16368 \mathrm{data}$. Data acquisition was controlled by Strawberry Tree software. Four electrodes were placed over left and right frontal (LF and RF), and left and right posterior (LP and RP) areas of the scalp with an additional reference electrode placed at the back of the head. EEG recording were taken at Day 7 posthatch and then each week for during 6 weeks. For additional details of the acquisition protocol see [1]. The recorded EEG signals are nonstationary and it also present artifacts due to saccadic eye movements [1]. In order to avoid these problems and facilitate a subsequent quantitative analysis, each signal was pre-processing using a methodology based on Wavelets (for procedure details see [4]) and summarized as follow: a) The Discrete Orthogonal Wavelet Transform of the signal is obtained considering $J_{\max }=-10$ wavelet resolution levels with spline cubic mother wavelet. After that a cleaning and stationary signal is obtained by reconstruction (inverse wavelet transform) using the resolution wavelet levels corresponding to the frequency range $0.5-32.0 \mathrm{~Hz}$. b) The wavelet frequency bands at witch the saccadic movement frequency appear, as well as, their time localization were identified. The corresponding wavelet coefficients were reduced in order that they present a contribution below the noise-signal level, and the corresponding frequency band is reconstructed. Finally the complete signal is obtained by superposition of the all wavelet reconstructed frequency bands (see Figs. 1 and 2 of [5]). 


\section{THE MULTIFRACTAL DETRENDED FLUCTUATION ANALYSIS}

Stanley and co-workers developed a method for the multifractal characterization of nonstationary time series [2], which is based on a generalization of the detrended fluctuation analysis (DFA). Following Ref. [2] the multifractal DFA (MFDFA) and how the scaling exponents are determined via the MFDFA method can be summarized as follow:

- Starting with a correlated time series (signal) $\{u(i), i=1, \cdots, N\}$ (where $N$ is the length of the series) the corresponding profile is determined by integration

$$
Y(k)=\sum_{i=1}^{k}[u(i)-<u>]
$$

where $\langle u\rangle=\frac{1}{N} \sum_{i=1}^{N} u(i)$ is the mean.

- The profile $Y(k)$ is cut into $N_{s} \equiv[N / s]$ nonoverlapping segments of equal length $s$. Since the record length $N$ need not be a multiple of the considered time scale $s$, a short part at the end of the profile will remain in most cases. In order not to disregard this part of the record, the same procedure is repeated starting from the other end of the record. Thus, $2 N_{s}$ segments are obtained altogether.

- The local trend for each segment $v=1, \cdots, 2 N_{S}$ is evaluated by least-square fit of the data. The detrended time series for segment duration $s$, denoted by $Y_{S}(i)$, is calculated as the difference between the original time series and the fits,

$$
Y_{S}(i)=Y(i)-p_{v}(i),
$$

where $p_{v}(i)$ is the fitting polynomial in the $v$-th segment. Since we use a polynomial fit of order $l$, we denote the algorithm as MFDFA- $l$. The detrending of the time series is done by subtraction of the fits from the profile, these methods differ in their capability of eliminating trends in the data. In $m$-th order of MFDFA, trends of order $m$ in the profile and $m-1$ in the original record are eliminated. Thus, a comparison of the results for different orders of MFDFA allows us to estimate the strength of the trends in the time series.

- For each of the $2 N_{S}$ segments the variance of the detrended time series $Y_{S}(i)$ is evaluated by averaging over all data point $i$ in the $v$-th segment

$$
F_{s}^{2}(v)=\frac{1}{s} \sum_{i=1}^{s}\left\{Y_{s}[(v-1) s+i]\right\}^{2} .
$$

- Average over all segments to obtain the $q$-th fluctuation function

$$
F_{q}(s)=\left\{\frac{1}{2 N_{s}} \sum_{v=1}^{2 N_{s}}\left[F_{s}^{2}(v)\right]^{q / 2}\right\}^{1 / q},
$$

where, in general, the index $q$ can take any real value. For $q=2$, the standard DFA procedure is retrieved.

- The scaling behavior of the fluctuation is determined by analyzing $\log \log$ plots $F_{q}(s)$ versus $s$ for each value of $q$. If the series $u(i)$ are long-range power-low correlated $F_{q}(s)$ increase, for large values of $s$, as a power-law

$$
F_{q}(s) \sim s^{h(q)} .
$$

For $q=0$ the value $h(0)$ cannot be determined directly using the average procedure in Eq. (4) because of the diverging exponent. Instead, using the L'Hôpital rule, a logarithmic average procedure has to be employed,

$$
F_{0}(s)=\exp \left\{\frac{1}{4 N_{s}} \sum_{v=1}^{2 N_{s}} \ln \left[F_{s}^{2}(v)\right]\right\} \sim s^{h(0)}
$$

For monofractal time series with compact support, $h(q)$ is independent of $q$, since the scaling behavior of the variance $F_{s}^{2}(v)$ is identical for all segments $v$, and the averaging procedure in Eq. (4) will give just this identical scaling behavior for all values of $q$. Only if small and large fluctuations scale differently, there will be a significant dependence of $h(q)$ on $q$ : If we consider positive values of $q$, the segments $v$ with large variance $F_{s}^{2}(v)$ will dominate the average $F_{q}(s)$. Thus, for positive values of $q, h(q)$ describes the scaling behavior of the segments with large fluctuations. On the contrary, for negative values of $q$, the segments $v$ with small variance $F_{s}^{2}(v)$ will dominate the average $F_{q}(s)$. Hence, 


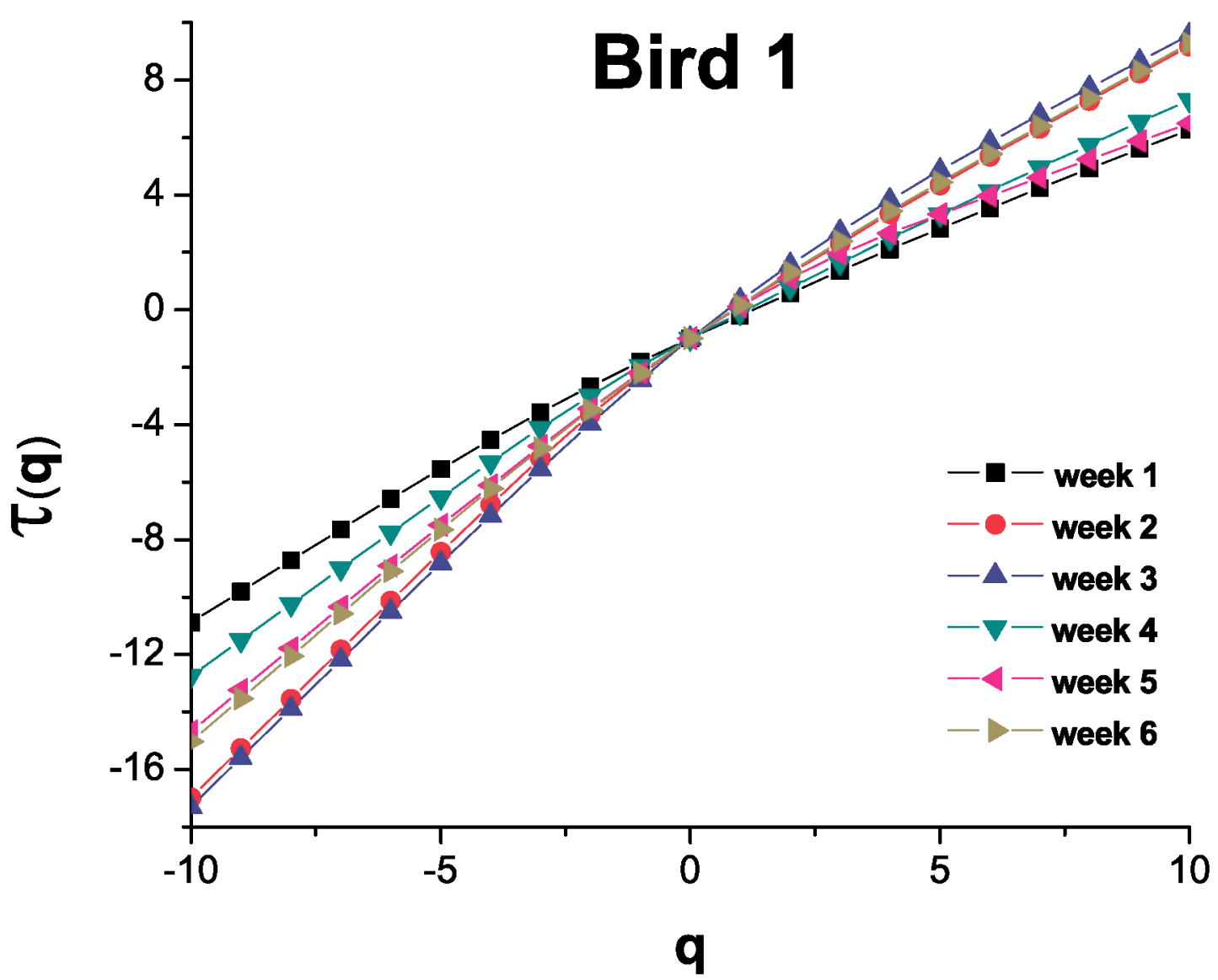

FIGURE 2. $\tau(q)$ corresponding to Bird $\# 1$ at frontal left electrode for the 6 weeks' posthatch.

for negative values of $q, h(q)$ describe the scaling behavior of the segments with small fluctuations.

Following Eqs. (4) and (6) and assume that the length $N$ of the series is an integer multiple of the scale $s$,

$$
\sum_{v}^{N / s}|Y(v s)-Y((v-1) s)|^{q} \sim s^{q} h(q)-1
$$

Stanley and co-workers show this multifractal formalism corresponding with the standard box counting theory, and they related both formalisms. It is obvious that the term $Y(v s)-Y((v-1) s)$ is identical to the sum of the numbers $u(i)$ within each segment $v$ of size $s$. This sum is the box probability $p_{s}(v)$ in the standard formalism for normalized series $u(i)$. The scaling exponent $\tau(q)$ is usually defined via the partition function $Z_{q}(s)$,

$$
Z_{q}(s) \equiv \sum_{v=1}^{N / s}\left|p_{s}(v)\right|^{q} \sim s^{\tau(q)}
$$

where $q$ is a real parameter as in the MFDFA. Then using the last equation and Eq. (8), they conclude that are identical and obtain the relation between the two sets of multifractal scaling exponents:

$$
\tau(q)=q h(q)-1
$$


Bird 1

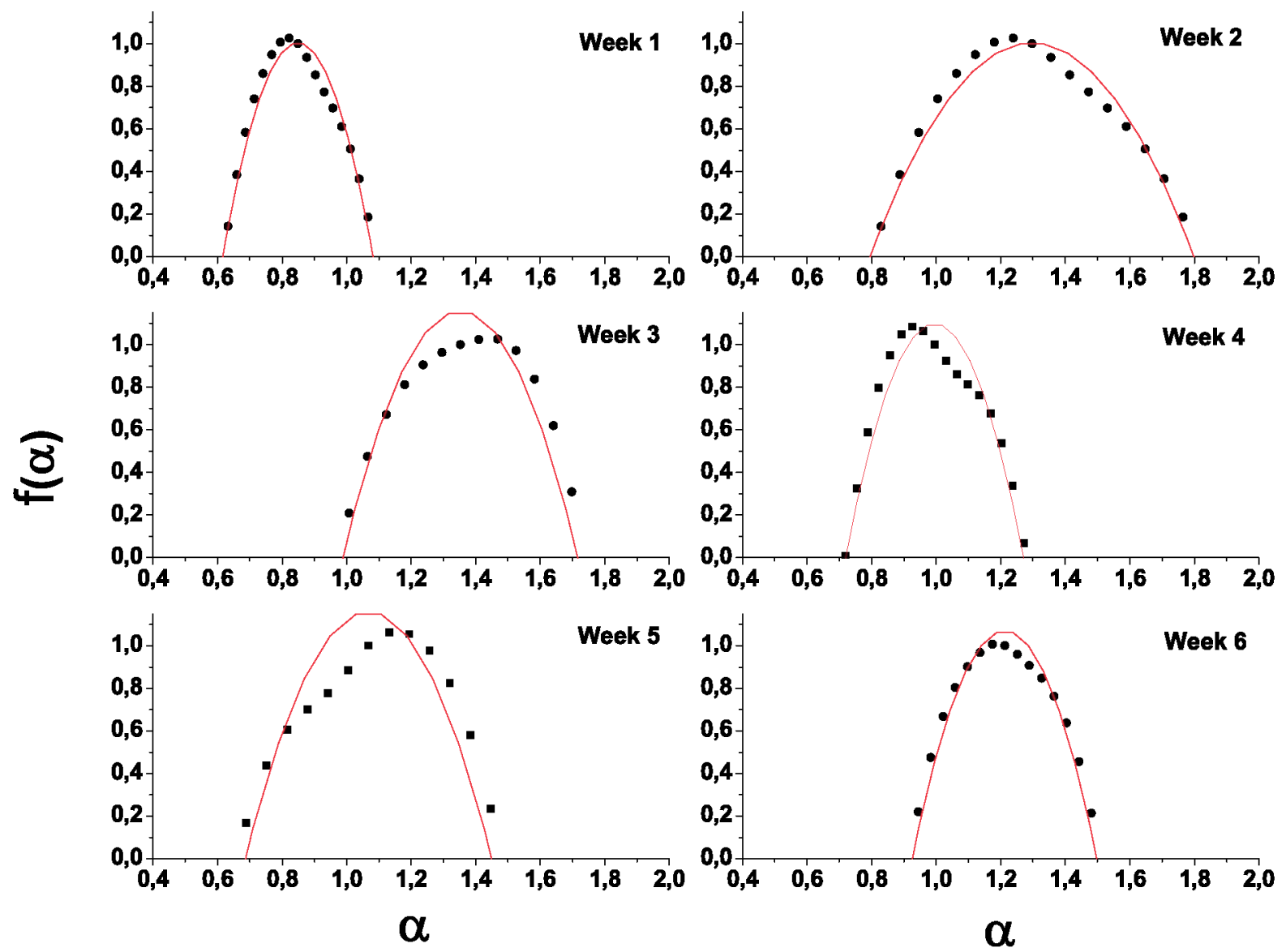

FIGURE 3. Multifractal spectrum corresponding to Bird \#1 at frontal left electrode for the 6 weeks' posthatch.

The singularity spectrum $f(\alpha)$ is another way to characterize the multifractality of the series. The parameter $\alpha$ is the Hölder exponent or the singularity intensity. The $f(\alpha)$ spectrum is related with $\tau(q)$ via a Legendre transformation $[8]$ :

$$
\alpha=\tau^{\prime}(q), \quad f(\alpha)=q \alpha-\tau(q) .
$$

Using Eq. (9) they related directly $\alpha$ and $f(\alpha)$ to $h(q)$ :

$$
\alpha=h(q)+q h^{\prime}(q), \quad f(\alpha)=q[\alpha-h(q)]+1
$$

\section{RESULTS AND DISCUSSION}

Synapse formation in the chicken brain occurs most rapidly around the time of hatching and is complete by 10 to 14 days posthatch [6]. Subsequently, the immature synapses and neurons gradually attain adult ultrastructural and biochemical properties. These changes occur in the period between 3 and 8 weeks posthatch in what has been termed the "maturation period" by Rostas, [7] during which neuronal circuits become fine-tuned. 
The EEG time series studied in the current work required pre-processing in order to eliminate undesired frequencies produced by a repetitive motion (saccadic movements) typical of the studied birds. This preprocessing is not straightforward (it can not be done with band pass filters) and was the object of a previous paper [4].

Starting from the cleaning EEG signals we calculate the fluctuation function $F(s)$ according with the equation Eq. (4) as function of the parameter $q$, varying between -10 and 10 , for each bird, week and channel. Then, sketching the function $F_{q}(s)$ versus $s$ in a graph with logarithmic scales, the functions $h(q)$ and $\tau(q)$ were obtained following Eqs. (6) and (8). Figure 1 shows the variation of $h(q)$ for Bird \#1 at the electrode LF for the six week posthatch recording. In the Fig. 2 the corresponding $\tau(q)$ functions are shown. Applying the Legendre relationships we obtain the multifractal spectrum $f(\alpha)$ as function of the singularities $\alpha$. This task was carried out on all the series, with the objective of comparing the multifractal spectrum for the different weeks. Figure 3 shows the evolution of the $f(\alpha)$ for the six weeks for the electrode LF corresponding to Bird \# 1. A clear difference in the position and shape of singularity spectrum is observed across the weeks.

Complex behavior of the signal is associated with the distribution of the singularities, $\alpha$. That is, points where the signal is continuous but not differentiable. In this case, Hölder exponents [9] describe the degree of regularity. If the measure of the set of points having the same regularity exponents is not an integer, it infers that the signal is fractal. For the multifractal case, we have a non-integer measure for different exponents $\alpha$. If the spectrum distribution $f(\alpha)$ is centered around $\alpha=1$ or for $\alpha>1(\alpha<1)$ the system is differentiable (non-differentiable) in the Hölder sense [9]. Then from Fig. 3 we can observe that the spectrum presents non-differentiable characteristics mainly in week 1 . From then the pattern starts to shift to the right side, and start to be more differentiable from weeks 3 to 6 . This behavior is associated with the "maturation period" described by Rostas a co-workers. Is interesting also to note that a wider spectrum appears at week 2 which can be associated with complete synapse formation. Another aspect that we can associated with the maturation period (weeks 3 to 6 ) is the asymmetry observed in the spectrum at weeks 3 to 5 . Note that it almost disappear at week 6 , when the neurological maturation of the bird is considered complete. We observed similar results with different birds and electrodes. The fractal characteristic does not present substantial changes at different electrodes. From the presents results we conclude that the MFDFA method can be used for the quantitative analysis of brain maturation change.

\section{ACKNOWLEDGMENTS}

This work was supported by The University of Newcastle through a research visitor grant to OAR and by Hunter Medical Research Institute and the NSW Department of Health. Also supported by Consejo Nacional de Investigaciones Científicas y Técnicas (CONICET) (PIP 5687/05) and Agencia Nacional de Promoción Cientifica y Tecnológica (PICT 20648/04), Argentina.

\section{REFERENCES}

1. M. Hunter, M. Battilana, T. Bragg, J. A. P. Rostas, Developmental Psychobiology 36, 23-28 (2000).

2. J. W. Kantelhardt, S. A. Zschiegner, E. Koscielny-Bunde, S. Havlin, A. Bunde and H. E. Stanley, Physica A 316, 87-114 (2002).

3. C. K. Peng, S. V. Buldyrev, A. L. Goldberger, S. Havlin, R. N. Mantegna, M. Simons, H. E. Stanley, Physica A 221 180-192 (1995).

4. A. Figliola, O. A. Rosso and E. Serrano, "Atenuación de frecuencias indeseadas usando transformada wavelet," in Proceedings XII RPIC, edited by Grupo de Electrónica Aplicada, Guillermo García Electrónica Edition, Río Cuarto, 2005 , pp. 28 -32.

5. J. G. Fernández, H. A. Larrondo, A. Figliola, E. Serrano, J. P. Rostas, M. Hunter and O. A. Rosso, "Brain maturation changes characterized by algorithmic complexity (Lempel and Zip complexity)," in present proceedings volume, edited by O. Descalzi, H. A. Larrondo and O. A. Rosso, AIP Conference Proceedings, American Institute of Physics, New York, 2007.

6. J. A. P. Rostas, J. M. Kavanagh, P. R. Dodd, J. W. Heath and D. A. Powis, Molecular Neurobiology 5, 203-215 (1992).

7. J. A. P. Rostas, "Molecular mechanisms of neuronal maturation: A model for synaptic plasticity," in Neural and behavioural plasticity: The use of the domestic chicken as a model, edited by R. J. Andrew, Oxford University Press, London, 1991, pp. $177-211$.

8. K. Falconer, Techniques in fractal geometry, John Wiley and Sons Ltd, New York, 1997.

9. S. Mallat, A wavelet tour of signal processing, 2nd Edition, American Press, San Diego, 1999. 